\title{
An ACO Algorithm with Adaptive Volatility Rate of Pheromone Trail
}

\author{
Zhifeng $\mathrm{Hao}^{1,2}$, Han Huang ${ }^{1}$, Yong Qin ${ }^{3}$, Ruichu Cai ${ }^{1}$ \\ ${ }^{1}$ College of Computer Science and Engineering, South China University of Technology, \\ Guangzhou 510640, P.R. China \\ ${ }^{2}$ National Mobile Communications Research Laboratory, Southeast University, Nanjing \\ 210096, P.R. China \\ ${ }^{3}$ Center of Information and Network, Maoming University, Maoming, Guangdong, 525000, \\ P.R. China \\ bssthh@163.com, hanhuang_scut@hotmail.com
}

\begin{abstract}
Ant colony optimization (ACO) has been proved to be one of the best performing algorithms for NP-hard problems as TSP. The volatility rate of pheromone trail is one of the main parameters in ACO algorithms. It is usually set experimentally in the literatures for the application of ACO. The present paper proposes an adaptive strategy for the volatility rate of pheromone trail according to the quality of the solutions found by artificial ants. The strategy is combined with the setting of other parameters to form a new ACO algorithm. Finally, the experimental results of computing traveling salesman problems indicate that the proposed algorithm is more effective than other ant methods.
\end{abstract}

Keywords. Ant colony optimization, pheromone trail, adaptive volatility rate

\section{Introduction}

ACO was first proposed by M. Dorigo and his colleagues as a multi-agent approach to deal with difficult combinatorial optimization problems such as TSP [1-2]. Since then, many applications to the NP-hard problems have shown the effectiveness of ACO [3].

The main parameters of ACO may conclude: $k$ is the number of artificial ants, $\rho$ is the parameter for volatility of pheromone trail and $\alpha, \beta$ determine the relative importance of pheromone value and heuristic information [2]. All of the parameters are usually set with experimental methods in the application of ACO [3]. The have been also several works [4-7] for the adaptive parameter setting of ACO algorithms.

This paper presents a tuning rule for $\rho$ based on the quality of the solution constructed by the artificial ant. Then, the adaptive rule is used to form a new ACO algorithm, which is tested to compute several benchmark TSP problems. The experimental result indicates that the proposed ACO algorithm with adaptive $\rho$ performs better than other ACO algorithms [2,8]. 


\section{Adaptive Volatility Rate of Pheromone Trail}

In ACO algorithms [3], each ant builds a tour (i.e. a feasible solution to the TSP problem) by repeatedly applying the state transition rule as Equation 1 shows.

$$
P_{g s}^{(m)}(t)= \begin{cases}\frac{\left[\tau_{g s}(t)\right]^{\alpha}\left[\eta_{g s}\right]^{\beta(g, t)}}{\sum_{r \in J_{m}(g)}\left[\tau_{g r}(t)\right]^{\alpha}\left[\eta_{g r}\right]^{\beta(g, t)}} & \text { if } s \in J_{k}(g) \\ 0 & \text { otherwise }\end{cases}
$$

where $P_{g s}^{m}$ is the probability with which the $m$-th ant moves from city $g$ to city $s$ in the $t$-th iteration, $\tau$ is the pheromone, $\eta=1 / d$ is the reciprocal of distance $d_{g s}$, and $J_{m}(g)$ is the set of cities not visited when ant $m$ is at city $g$.

After constructing its tour, an artificial ant also modifies the amount of pheromone on the visited edges by applying the pheromone updating rule:

$$
\tau_{g s}(t+1)=(1-\rho) \tau_{g s}(t)+\rho \Delta \tau_{g s}(t)
$$

where $\Delta \tau_{g s}(t)$ is the increment for the pheromone of edge $(g, s)$ at the $t$-th iteration, and $\rho=0.1$ is the volatility rate of the pheromone trail [2-3].

In order to update the pheromone according to the quality of solutions found by ants, an adaptive rule for volatility of the pheromone trail is designed as follows:

$$
\rho_{m}=L_{m}^{-1} /\left(L_{m}^{-1}+L_{P}^{-1}\right)
$$

where $L_{m}$ is the length of the solution $S_{m}$ found by the $m$-th ant, and $L_{P}$ is the length of the solution built based on the pheromone matrix (Equation 4).

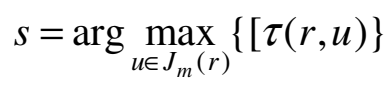

where $s$ is the city selected as the next one to city $r$ for any $(r, s) \in S_{P}$.

The motivation of the proposed rule is: better solutions should contribute more pheromone, and the worse ones contribute less. We use this rule to design a new ACO algorithm, which is similar to ant colony system (ACS) [2] except for the updating rule [2] (Equation 5). For $i=1, \ldots, k+1$ ( $k$ ants and the best-so-far ant),

$$
\tau_{g s}(t+1)=\left(1-\rho_{i}\right) \tau_{g s}(t)+\rho_{i} L_{i}^{-1}
$$

where $\forall(g, s) \in S_{i}$ and $\rho_{i}=L_{i}^{-1} /\left(L_{i}^{-1}+L_{P}^{-1}\right)$ for the $t$-th iteration. 


\section{Numerical Results}

This section indicates the numerical results in the experiment that the proposed ACO algorithm is used to solve TSP problems [9]. Other ant approaches ACS [2] and the ACO [8] are also tested in the same machines as the comparison with the proposed ACO. Several TSP instances are computed by the three algorithms on a PC with an Intel Pentium 550MBHz Processor and 256MB SDR Memory, and the results are shown in Table 1. It should be noted that every instance is computed 20 times. The algorithms would not stop until a better solution could be found in 500 iterations.

Table 1 shows that the proposed ACO algorithm (PACO) performs better than ACS [2] and the ACO [8]. The shortest lengths and the average lengths obtained by PACO are shorter than those found by ACS and the ACO in all of the TSP instances. Furthermore, it can be concluded that the standard deviations of the tour lengths obtained by PACO are smaller than those of another algorithms. Therefore, we can conclude that PACO is proved to be more effective and steady than ACS [2] and the ACO [8]. Computation time cost of PACO is not less than ACS and ACO in all of the instances because it needs to compute the value of volatility rate $k+1$ times per iteration. Although all optimal tours of TSP problems cannot be found by the tested algorithms, all of the errors for PACO are much less than that for another two ACO approaches. The algorithms may make improvement in solving TSP when reinforcing heuristic strategies like ACS-3opt [2] are used.

Table 1. Comparison of the results in TSP instances

\begin{tabular}{|c|c|c|c|c|c|}
\hline Problem & Algorithm & best & Ave & time $(\mathrm{s})$ & Standard deviation \\
\hline \multirow{3}{*}{ kroA100 } & ACS & 21958 & 22088.8 & 65 & 1142.77 \\
\hline & $\mathrm{ACO}$ & 21863 & 22082.5 & 94.6 & 1265.30 \\
\hline & PACO & 21682 & 22076.2 & 117.2 & 549.85 \\
\hline \multirow{3}{*}{ ts 225} & ACS & 130577 & 133195 & 430.6 & 7038.30 \\
\hline & $\mathrm{ACO}$ & 130568 & 132984 & 439.3 & 7652.80 \\
\hline & PACO & 130507 & 131560 & 419.4 & 1434.98 \\
\hline \multirow{3}{*}{ pr226 } & $\mathrm{ACS}$ & 84534 & 86913.8 & 378.4 & 4065.25 \\
\hline & $\mathrm{ACO}$ & 83659 & 87215.6 & 523.8 & 5206.70 \\
\hline & PACO & 81967 & 83462.2 & 762.2 & 3103.41 \\
\hline \multirow{3}{*}{$\operatorname{lin} 105$} & ACS & 14883 & 15125.4 & 88.8 & 475.37 \\
\hline & $\mathrm{ACO}$ & 14795 & 15038.4 & 106.6 & 526.43 \\
\hline & PACO & 14736 & 14888 & 112.2 & 211.34 \\
\hline \multirow{3}{*}{ kroB 100} & $\mathrm{ACS}$ & 23014 & 23353.8 & 56.2 & 685.79 \\
\hline & $\mathrm{ACO}$ & 22691 & 23468.1 & 102.9 & 702.46 \\
\hline & PACO & 22289 & 22728 & 169.6 & 668.26 \\
\hline \multirow{3}{*}{ kroC100 } & $\mathrm{ACS}$ & 21594 & 21942.6 & 54.8 & 509.77 \\
\hline & $\mathrm{ACO}$ & 21236 & 21909.8 & 78.1 & 814.53 \\
\hline & PACO & 20775 & 21598.4 & 114.8 & 414.62 \\
\hline \multirow{3}{*}{$\operatorname{lin} 318$} & ACS & 48554 & 49224.4 & 849.2 & 1785.21 \\
\hline & $\mathrm{ACO}$ & 48282 & 49196.7 & 902.7 & 2459.16 \\
\hline & PACO & 47885 & 49172.8 & 866.8 & 1108.34 \\
\hline
\end{tabular}




\section{Discussions and Conclusions}

This paper proposed an adaptive rule for volatility rate of pheromone trail, attempting to adjust the pheromone based on the solutions obtained by artificial ants. Thus, a new ACO algorithm is designed with this tuning rule. There is a special pheromone updating rule in the proposed algorithm whose framework is similar to Ant Colony System. There are some experimental comparisons among the proposed ACO approach and other methods [2,8] in solving TSP problems. The results show the effectiveness of the proposed algorithm.

Further study is suggested to explore the better management for the optimal setting of the parameters of ACO algorithms, which will be very helpful in the application.

Acknowledgements. This work has been supported by the National Natural Science Foundation of China (60433020, 10471045), Program for New Century Excellent Talents in University (NCET-05-0734), Natural Science Foundation of Guangdong Province (031360, 04020079), Excellent Young Teachers Program of Ministry of Education of China, Fok Ying Tong Education Foundation (91005), Key Technology Research and Development Program of Guangdong Province (2005B10101010), State Key Lab. for Novel Software Technology, Nanjing University (200603), open research fund of National Mobile Communications Research Laboratory, Southeast University (A200605), Nature Science Foundation of Guangdong (05011896), and Nature Science Foundation of Education Department of Guangdong Province (Z03080).

\section{References}

1. Dorigo, M., Caro, G.D., Gambardella, L.M.: Ant algorithms for Discrete Optimization, Massachusetts Institute of Technology, Artificial Life 5: 137-172, 1999

2. Dorigo, M., Gambardella, L.M.: Ant Colony System: A Cooperative Learning Approach to the Travelling Salesman Problem, IEEE Transactions on Evolutionary Computation, 1 (1), 53-66, 1997

3. Dorigo, M., Stützle, T.: Ant Colony Optimization. MIT Press, Cambridge, MA (2004)

4. Watanabe, I., Matsui, S.L.: Improving the Performance of ACO Algorithms by Adaptive Control of Candidate Set, Evolutionary Computation, 2003. CEC '03. The 2003 Congress on Volume 2, 8-12 Dec. 2003 Page(s): 1355 - 1362 Vol.2

5. Pilat, M.L., White, T.: Using Genetic Algorithms to Optimize ACS-TSP, M. Dorigo et al. (Eds.):ANTS 2002, LNCS 2463, 282-287, 2002

6. Gambardella, L.M., Dorigo, M.: Ant-Q: A Reinforcement Learning approach to the travelling salesman problem, Appeared in: Proceedings of ML-95, Twelfth Intern. Conf. On Machine Learning, Morgan Kaufmann, 252-260, 1995

7. Huang H., Yang X.W., Hao Z.F., Cai R.C.: A novel ACO algorithm with adaptive parameter, D.S. Huang et al. (Eds.): ICIC 2006, LNBI 4115, 12-21, 2006

8. Sun, J., Xiong, S.W., Guo, F.M.: A new pheromone updating strategy in ant colony optimization, Proceedings of 2004 International Conference on Machine Learning and Cybernetics, 1, 620-625, 2004

9. Reinelt, G.: TSPLIB. A traveling salesman problem library. ORSA Journal on Computing, 3 (4), 376-384, 1991 